Agrotrópica 27(3): 289 - 296. 2015.

Centro de Pesquisas do Cacau, Ilhéus, Bahia, Brasil

\title{
MANEJO DA FERTILIDADE DO SOLO EM REFLORESTAMENTO DE CLAREIRAS DA FLORESTA AMAZÔNICA
}

\author{
Bruna Trovo Canizella, Adônis Moreira \\ ${ }^{1}$ Universidade Estadual de Londrina, 86055-900, Londrina, Paraná, Brasil; ${ }^{2}$ Embrapa soja, 86001-970, Londrina, Paraná, \\ Brasil, adonismoreira66@gmail.com.
}

\begin{abstract}
$\mathrm{Na}$ Amazônia, a retirada da floresta primária com a posterior remoção da camada superficial do solo acarreta na diminuição na quantidade de nutrientes disponíveis do solo. Nesse local, com o posterior reflorestamento, o crescimento das plantas é lento mesmo com aplicação de fertilizantes. O objetivo deste trabalho foi estudar os efeitos da adubação com compostagem de resíduos provenientes da roçagem dos jardins e mineral (N-P-K) sobre os atributos químicos do solo na revegetação de clareiras abertas na região central da Amazônia tropical úmida. Foram avaliados os atributos químicos do solo $\left[\mathrm{pH}\left(\mathrm{H}_{2} \mathrm{O}\right)\right.$, matéria orgânica, $\mathrm{P}, \mathrm{K}, \mathrm{Ca}^{2+}, \mathrm{Mg}^{2+}, \mathrm{Al}^{3+}, \mathrm{H}^{+}+\mathrm{Al}^{3+}, \mathrm{Cu}, \mathrm{Fe}$, $\mathrm{Mn}$ e $\mathrm{Zn}]$ em duas florestas primárias e três clareiras reflorestadas com idades distintas (5, 10 e 15 anos) em diferentes profundidades $(0-10$ e $11-30 \mathrm{~cm})$. Os resultados mostraram que solo mesmo com aplicação de nutrientes, apresenta difícil reposição da fertilidade natural. Após 15 anos de revegetação a fertilidade do solo não apresentou níveis de nutrientes semelhantes ao da floresta primária. A aplicação de $2 t$ ha $^{-1}$ de calcário 30 dias antes do plantio e dois litros de compostos orgânico, $200 \mathrm{~g}$ do adubo N-P-K (10-30-10) e $50 \mathrm{~g}$ de $\mathrm{MgSO}_{4}$ na cova de plantio não foi suficiente para o restabelecimento da floresta nas clareiras.
\end{abstract}

Palavras-chave: Matéria orgânica, balanço de íons no solo, eficiência de uso de fertilizantes.

Management of soil fertility in the recovery of clearing of Amazon rainforest. In the Amazon rainforest, with the removal of primary forest with the subsequent removal of topsoil to the exploration of areas leads to significant decrease of nutrients. The subsequent reforestation of these areas, the plant growth is slow even with the application of fertilizers. The aim of this study was to evaluate the effects of fertilization with waste composting and mineral (NPK) on soil chemical properties in reforestation of clearings in the central region of humid tropical Amazon. We evaluated two primary forests and three recovering clearing with 5, 10, and 15-yearold in different depths (0-10 and 11-30 cm). The soil chemical properties [pH $\left(\mathrm{H}_{2} \mathrm{O}\right)$, organic matter, $\mathrm{P}, \mathrm{K}, \mathrm{Ca}^{2+}, \mathrm{Mg}^{2+}$, $\mathrm{Al}^{3+}, \mathrm{H}^{+}+\mathrm{Al}^{3+}, \mathrm{Cu}, \mathrm{Fe}, \mathrm{Mn}$, and $\mathrm{Zn}$ ] were evaluated. The results showed that soil has management fragility with low fertility. After 15-years-old of planting was not enough to restore the soil fertility levels of primary forest. The $2 \mathrm{tha}$ ${ }^{1}$ of lime application 30 days before planting and two liters of organic compounds, $200 \mathrm{~g}$ of N-P-K (10-30-10), and 50 $\mathrm{g}$ of $\mathrm{MgSO}_{4}$ in the planting hole was not enough to restore forest in the clearings.

Key words: organic matter, ions balance in the soil, fertilizer use-efficiency. 


\section{Introdução}

Projetos de assentamento com exploração madeireira, queima da floresta primária ou secundária para introdução de pastagens ou culturas de subsistência e exploração de minérios, petróleo e/ou gás natural têm acarretado pressão considerável sobre ecossistema regional na Amazônia (Fearnside, 1999; Santos Júnior et al., 2006). Fato esse verificado na província petrolífera Geólogo Pedro de Moura (BOGPM), localizada às margens do rio Urucu, Município de Coari, Estado do Amazonas, onde são encontradas áreas alteradas para a prospecção de gás natural e petróleo, nas quais são abertas clareiras e retirada grandes quantidades de terra para prospecção e construção de estradas que são utilizadas na manutenção dos dutos e infraestrutura (Moreira e Costa, 2004).

Após a remoção, parte da camada superficial é retornada ao local, no qual é feito, o reflorestamento com espécies nativas da região (Moreira e Costa, 2004; Prudente et al., 2010). Com esta prática de manejo, muitas plantas não apresentam crescimento satisfatório ou morrem ficando o solo sem cobertura vegetal, predisposto às intempéries climáticas com consequente erosão (Rodrigues et al., 2006).

Independentemente do reflorestamento ou não, a retirada de solo para atividades de extração de gás tem causado impacto negativo neste ecossistema, visto que os solos, apesar de estarem sob floresta densa, são caracterizados pela baixa fertilidade natural e dependência do fluxo contínuo de serapilheira e nutrientes no sistema (Stark, 1970; Sanchez e Salinas, 1981; Moreira \& Fageria, 2009. Nestes locais, o equilíbrio da fertilidade é mantido pelos processos biogeoquímicos que atuam na formação e dinâmica da matéria orgânica do solo (Malavolta, 1987), e responsável pela manutenção da floresta em estágio clímax (Poggiani \& Schumacher, 2004).

A consequência deste manejo é que com a remoção da floresta esse ciclo é quebrado acarretando a rápida mineralização da matéria orgânica (MO) ocasionado, principalmente, pela diminuição do material vegetal senescente, como folhas, galhos e frutos (Malavolta, 1987; Moreira e Costa, 2004).

O objetivo deste trabalho foi verificar os efeitos do manejo do solo e da adubação com composto orgânico e mineral N-P-K (10-30-10) utilizada na recuperação das clareiras em áreas para prospecção de petróleo e gás natural na restituição da fertilidade após 5, 10 e 15 anos do plantio de mudas de espécies arbóreas nativas da região sobre os atributos químicos do solo na região central da Amazônia tropical úmida.

\section{Material e Métodos}

As amostras de solo foram coletadas em cinco áreas de "terra firme" (local sem influência dos rios) localizadas na BOGPM - Base Operacional Geólogo Pedro de Moura (PETROBRAS-BR ${ }^{\circledR}$ ), situada nas coordenadas geográficas (453’7,33" LS; 65²0'59,99" LW), município de Coari, Estado do Amazonas.

Nessa região, os solos são constituídos por sedimentos da formação Solimões, apresentando relevo ondulado e floresta tropical densa (Lima Filho et al., 2001), sendo classificados como Plintossolos, Latossolos, Gleissolos eArgissolos (Vieira e Santos, 1987), com predominância de Argissolos na BOGPM. O clima da região é o tropical úmido pela classificação de Köppen apresentando ciclo sazonal de distribuição de precipitação bem característico, alternando uma estação seca de junho a novembro e estação chuvosa de dezembro a maio. A média anual de precipitação é de $2.239 \mathrm{~mm}$, com $66,1 \%$ distribuídas na estação chuvosa e $33,9 \%$ na estação seca. As temperaturas médias variam de $25,2^{\circ} \mathrm{C}$ a 26,2 ${ }^{\circ} \mathrm{C}$ ao ano. (Arruda, 2005; Prudente et al., 2010). A região é basicamente composta por dois grandes grupos de vegetação: floresta ombrófila densa de 'terra firme' (representando 80 a $85 \%$ da cobertura vegetal) e 'várzeas' permanentes ou periodicamente inundadas (Prudente et al., 2010; Santos Júnior et al., 2006).

Uma cronossequência formada por duas florestas primárias e três clareiras com diferentes idades de reflorestamento (5, 10 e 15 anos) com as seguintes espécies nativas da região produzidas via seminal: lacre (Vismia guinensis), ingá (Inga edulis Mart.), Angelimpedra (Dinizia excelsa), goiaba-de-anta (Bellucia dichotoma Triana), jamelão [Eugenia cumini (L.) Druce], sapota [Chrysophyllum sanguinolentum (Piere) Baehni], embaúba (Cecropia ficifolia Smeth), angico (Abarema piresii), embaubão (Cecropia sciadophylla Mart) e sucuúba (Himantanthus sucuuba) plantadas ao acaso, simulando uma dispersão florestal. 
O manejo de recuperação e replantio da cobertura vegetal nas clareiras foi baseado na correção da acidez do solo com aplicação de duas toneladas de calcário dolomítico (PRNT 85\%) em área total para atingir a saturação por bases (V\%) a 50\% e adubação de plantio realizada 30 dias antes do plantio das mudas na cova (no início do período das chuvas na região) com dois litros de composto orgânico proveniente da usina de tratamento de resíduo da BOGPM [composição química média: $38 \mathrm{mg} \mathrm{kg}^{-1}$ de Cu, $158 \mathrm{mg} \mathrm{kg}^{-1}$ de Fe, $60 \mathrm{mg} \mathrm{kg}$ ${ }^{1}$ de $\mathrm{Mn}$ e $110 \mathrm{mg} \mathrm{kg}^{-1}$ de Zn (Uchoa et al., 2006)]. Foi também aplicado $200 \mathrm{~g}$ do formulado 10-30-10 (N$\left.\mathrm{P}_{2} \mathrm{O}_{5}-\mathrm{K}_{2} \mathrm{O}\right)$ e $50 \mathrm{~kg}$ de $\mathrm{MgSO}_{4}(55 \%$ de $\mathrm{Mg}$ ) feitas em covas de $40 \times 40 \times 40 \mathrm{~cm}$ e em cobertura $(50 \pm 5 \mathrm{~cm})$ ao redor das plantas (Santos Júnior et al., 2006).

Em cada clareira (áreas 18, 21 e 22) e na floresta primária (áreas 15 e 10) foram coletadas dez amostras de solo nas entrelinhas de plantio com trado tipo holandês nas profundidades $(0-10 \mathrm{~cm}$ e $11-30 \mathrm{~cm})$. Posteriormente, estas foram secas ao ar (TFSA), passadas em peneiras de $2,0 \mathrm{~mm}$ e levadas ao laboratório para determinação do $\mathrm{pH}$ (água), $\mathrm{P}$ e K disponível (extrator Mehlich 1), $\mathrm{Ca}^{2+}, \mathrm{Mg}^{2+}$ e $\mathrm{Al}^{3+}$ trocável $\left(\mathrm{KCl} \mathrm{1,0} \mathrm{mol} \mathrm{L}^{-1}\right), \mathrm{H}^{+}+\mathrm{Al}^{3+}$ (acetato de cálcio 1,0 $\mathrm{mol} \mathrm{L}^{-1}$ ) e $\mathrm{Cu}, \mathrm{Fe}, \mathrm{Mn}$ e Zn disponível (Mehlich 1), CTC a pH 7,0 e matéria orgânica (MO) do solo, conforme metodologias descritas pela Embrapa (1997).

Para verificar o equilíbrio iônico do $\mathrm{K}^{+}, \mathrm{Ca}^{2+}{\mathrm{e} \mathrm{Mg}^{2+}}^{2+}$ do solo, foi determinado as relações $\mathrm{Ca}^{2+} / \mathrm{Mg}^{2+}, \mathrm{Mg}^{2+} /$ $\mathrm{K}^{+}, \mathrm{Ca}^{2+} / \mathrm{K}^{+}$e $\mathrm{K}^{+} /\left(\mathrm{Ca}^{2+}+\mathrm{Mg}^{2+}\right)$ e o balanço de íons na CTC do solo de acordo com de acordo com Tomé Júnior (1997).

Os dados foram tabulados para verificação da distribuição normal (média, mediana, desvio padrão, curtose e assimetria) e homogeneidade de variâncias. Devido à ausência de faixas de interpretação da disponibilidade de nutrientes no solo para o Estado do Amazonas, os valores encontrados foram comparados com as faixas de suficiência de fertilidade indicadas por Ribeiro et al. (1999) para o Estado de Minas Gerais.

\section{Resultado e Discussão}

Nas Tabelas 1, 2 e 3 são apresentados os resultados dos atributos químicos do solo nas profundidades de 0-10 cm e 11-30 cm das três áreas reflorestadas [clareiras 18 (10 anos), 21 (5 anos) e 22 (15 anos)] e nas duas florestas primárias (áreas 10 e 15). Para as profundidades, ano de plantio das espécies florestais nas clareiras e do teor de MO, a aplicação dos fertilizantes e corretivos da acidez não acarretaram em aumento dos teores de $\mathrm{P}, \mathrm{K}^{+}, \mathrm{Ca}^{2+}$ e $\mathrm{Mg}^{2+}$ do solo para níveis próximos dos considerados adequados, independentemente do tipo cultura instalada (Ribeiro et al., 1999). De acordo Pezarico et al. (2013), ambientes mais afetados quanto à qualidade do solo indicam tendências à degradação ambiental, visto que mesmo com a introdução e diversificação no plantio com espécies nativas da região, que apresentam diferentes características sucessionais nas clareiras, na floresta primária, devido às condições clímax, existem condições mais favoráveis para a melhoria e manutenção da qualidade química e biológica do solo (Poggiani \& Schumacher, 2004).

Na profundidade de 0-10 cm e $11-30 \mathrm{~cm}$, exceto os teores de $\mathrm{MO}, \mathrm{Ca}^{2+}, \mathrm{Fe}$ e $\mathrm{Mn}$ no solo, os valores de $\mathrm{pH}, \mathrm{P}, \mathrm{K}^{+}, \mathrm{Mg}^{2+}, \mathrm{H}^{+}+\mathrm{Al}^{3+}, \mathrm{Cu}$ e $\mathrm{Zn}$ apresentaram valores de média e mediana semelhantes e coeficientes de assimetria e curtose considerados próximos de zero (Tabela 1), o que indica que a distribuição dessas variáveis se assemelha a uma normal (Freitas et al., 2008), ou seja, não houve variabilidade do manejo adotado. No entanto, os altos coeficientes de variação verificados nos atributos químicos do solo (Tabela 1) corroboram Coelho (2004) sobre a alta variabilidade existente entre os cátions trocáveis e a MO do solo, possivelmente decorrente das mudanças intrínsecas dos processos de formação da camada superficial do solo com a incorporação de diferentes resíduos vegetais (folhas, sementes, galhos, etc.) dentro dos anos de plantio ocasionados pelo balanço existente entre o tempo de reposição florestal e a área que está sendo coberta durante desenvolvimento das plantas (Moreira e Costa, 2004).

Nas condições estudadas, devido à grande disponibilidade de resíduos orgânicos proveniente da compostagem feita na BOGPM, a aplicação durante vários anos de material orgânico diminuiria esta variação, visto que o aumento da quantidade aplicada no plantio das mudas e em cobertura até a formação da copa seria uma alternativa viável nas condições estudadas e ocasionaria em maior economia no uso de fertilizantes químicos Moreira \& Gonçalves, 2006; Arruda et al., 2012). 
Tabela 1. Análise descritiva das propriedades químicas do solo sob floresta primária e clareiras reflorestadas

\begin{tabular}{|c|c|c|c|c|c|c|c|c|c|c|c|}
\hline \multirow[b]{2}{*}{ Medidas } & \multirow{2}{*}{$\begin{array}{l}\mathrm{pH} \\
\mathrm{H}_{2} \mathrm{O}\end{array}$} & $\mathrm{P}$ & $\mathrm{K}^{+}$ & $\mathrm{Ca}^{2+}$ & $\mathrm{Mg}^{2+}$ & $\mathrm{H}^{+}+\mathrm{Al}^{3+}$ & \multirow{2}{*}{$\begin{array}{l}\text { MO } \\
\mathrm{g} \mathrm{dag}^{-1}\end{array}$} & $\mathrm{Cu}$ & $\mathrm{Fe}$ & $\mathrm{Mn}$ & \multirow{2}{*}{$\mathrm{Zn}$} \\
\hline & & \multicolumn{2}{|c|}{$---\mathrm{mg} \mathrm{dm}^{-3}----$} & \multicolumn{3}{|c|}{------ $\mathrm{cmol}_{\mathrm{c}} \mathrm{dm}^{-3}$} & & \multicolumn{3}{|c|}{--------------mg dm ${ }^{-3}$-------------- } & \\
\hline \multicolumn{12}{|c|}{$0-10 \mathrm{~cm}$} \\
\hline Média & 4,8 & 2 & 32,2 & 0,47 & 0,08 & 8,8 & 26,1 & $0, \overline{33}$ & 115 & 0,86 & 0,76 \\
\hline Mediana & 4,6 & 2 & 30 & 0,04 & 0,08 & 10,3 & 16,8 & 0,3 & 92 & 0,91 & 0,69 \\
\hline Mínimo & 4 & 1 & 21 & 0,03 & 0,03 & 3 & 9,2 & 0,21 & 21 & 0,17 & 0,44 \\
\hline Máximo & 6,6 & 4 & 43 & 2,13 & 0,17 & 11,3 & 49,2 & 0,63 & 217 & 1,51 & 1,31 \\
\hline Assimetria & 1,12 & 1,08 & 0,14 & 1,67 & 1,31 & $-0,42$ & 0,46 & 0,48 & 0,23 & 0,23 & 0,95 \\
\hline Curtose & 0,06 & 0,08 & $-0,2$ & 1,15 & 0,53 & 0,61 & $-2,05$ & 0,82 & $-2,06$ & $-2,1$ & $-0,26$ \\
\hline Desvio Padrão & 0,99 & 1,2 & 9,4 & 0,86 & 0,05 & 3,2 & 17,5 & 0,16 & 84,89 & 0,6 & 0,33 \\
\hline $\mathrm{CV} \%$ & 20,4 & 57,7 & 29 & 174,9 & 65,8 & 30,4 & 66,8 & 48,4 & 73,2 & 69,4 & 42,7 \\
\hline Shapiro Wilk & ns & ns & ns & $*$ & ns & ns & $*$ & ns & $*$ & $*$ & $\mathrm{~ns}$ \\
\hline \multicolumn{12}{|c|}{$11-30 \mathrm{~cm}$} \\
\hline Média & 4,8 & 0,6 & 25,4 & 0,08 & 0,05 & 8,6 & 13,1 & 0,29 & 77,8 & 8,83 & 0,42 \\
\hline Mediana & 4,9 & 0,4 & 22 & 0,04 & 0,04 & 8,3 & 5,8 & 0,31 & 49 & 0,5 & 0,4 \\
\hline Mínimo & 3,9 & 0,4 & 20 & 0,02 & 0,01 & 4,8 & 3,4 & 0,2 & 9 & 0,13 & 0,37 \\
\hline Máximo & 5,9 & 1 & 40 & 0,21 & 0,09 & 12,8 & 29 & 0,37 & 233 & 42 & 0,54 \\
\hline Assimetria & 0,27 & 0,48 & 1,7 & 0,27 & 0,3 & 0,3 & 0,65 & $-0,2$ & 1,34 & 1,78 & 1,15 \\
\hline Curtose & $-0,33$ & $-0,28$ & 1,26 & 1,18 & $-0,65$ & $-0,21$ & $-1,65$ & 1 & 0,47 & 1,4 & $-0,01$ \\
\hline Desvio Padrão & 0,8 & 0,31 & 7,76 & 0,07 & 0,03 & 2,7 & 10,8 & 0,06 & 86,6 & 17,49 & 0,07 \\
\hline $\mathrm{CV} \%$ & 17 & 48,4 & 30,6 & 94 & 68,9 & 31,7 & 82,4 & 21,3 & 111,3 & 197,9 & 15,8 \\
\hline Shapiro Wilk & ns & ns & $\mathrm{ns}$ & $*$ & ns & $\mathrm{ns}$ & $*$ & ns & $*$ & $*$ & ns \\
\hline \multicolumn{12}{|c|}{ Média das profundidades } \\
\hline Média & 4,8 & 1,3 & 28 & 0,3 & 0,06 & 8,7 & 19,6 & 0,31 & 96,4 & 4,85 & 0,59 \\
\hline Mediana & 4,4 & 1 & 24 & 0,04 & 0,06 & 9 & 14,7 & 0,31 & 65,5 & 0,71 & 0,48 \\
\hline Mínimo & 3,9 & 0,4 & 20 & 0,02 & 0,01 & 3 & 3,4 & 0,2 & 9 & 0,13 & 0,37 \\
\hline Máximo & 6,6 & 4 & 43 & 2,13 & 0,13 & 12,8 & 49,2 & 0,63 & 233 & 42 & 1,31 \\
\hline Assimetria & 0,87 & 1,78 & 0,78 & 2,95 & 1,48 & $-0,75$ & 0,95 & 0,1 & 1,73 & 3,15 & 1,9 \\
\hline Curtose & 0,01 & 0,5 & $-0,39$ & 9,15 & 2,96 & 0,25 & $-0,31$ & 0,33 & $-1,29$ & 9,95 & 0,66 \\
\hline Desvio Padrão & 0,91 & 1,1 & 9,32 & 0,65 & 0,05 & 3 & 16,9 & 0,12 & 87,6 & 13,07 & 0,28 \\
\hline $\mathrm{CV} \%$ & 18,9 & 84 & 32,4 & 217,3 & 74 & 34,2 & 81,9 & 40,1 & 90,9 & 269,6 & 49,7 \\
\hline Shapiro Wilk & $\mathrm{ns}$ & ns & ns & $*$ & ns & ns & $*$ & ns & $*$ & $*$ & ns \\
\hline
\end{tabular}

*significativo a $5 \%$ de probabilidade. ${ }^{\text {ns }}$ ão significativo.

A acidez potencial $\left(\mathrm{H}^{+}+\mathrm{Al}^{3+}\right)$ permaneceu elevada (Ribeiro et al., 1999), independente da profundidade e do local amostrado (Tabela 2), mesmo assim, houve redução de $73 \%$ dos níveis até cinco anos do plantio das mudas, possivelmente em função dos efeitos da calagem, que apesar de não ter atingido os $50 \%$ da saturação por base, ocasionou em elevação a níveis próximos da floresta primária (Tabela 2). Os teores elevados do $\mathrm{H}^{+}+\mathrm{Al}^{3+}$ verificados corroboram Demattê (1988) e Moreira \& Fageria, 2009, ao reportarem, que mesmo sob floresta primária e diferentes tipos de manejo, os solos da região central da Amazônia apresentam carência de bases trocáveis, sendo os sítios de troca ocupados quase que exclusivamente por estes dois íons. No caso do pH, semelhante a acidez potencial, a alteração provavelmente foi devida a quantidade de calcário aplicada e elevação do teor de MO verificado do plantio mais novo (5 anos) para o mais antigo (15 anos), o que acarreta em possível elevação do poder tampão, o que pode dificultar a mudança de faixa de $\mathrm{pH}$ do solo (Raij, 1997).

No caso da MO, como verificado por Moreira e Costa (2004) nas mesmas condições de manejo, mesmo com a elevação dos teores nas duas profundidades amostradas, os valores ficaram $243,6 \%$ inferiores a média das duas florestas primárias, mesmo após 15 anos de plantio das árvores para a reposição florestal (Tabela 2), indicando que os efeitos negativos com a 
Tabela 2. Atributos químicos do solo (MOS) de áreas alteradas (clareiras) e da floresta primária na BOGPM. Município de Coari, AM

\begin{tabular}{|c|c|c|c|c|c|c|c|}
\hline Clareiras/Profundidade & $\begin{array}{l}\mathrm{pH} \\
\text { água }\end{array}$ & $\begin{array}{l}\mathrm{P} \\
---\mathrm{mg}\end{array}$ & $\begin{array}{r}\mathrm{K} \\
\mathrm{m}^{-3}----\end{array}$ & $\mathrm{Ca}^{2+}$ & $\begin{array}{c}\mathrm{Mg}^{2} \\
-\mathrm{cmol}_{\mathrm{c}} \mathrm{dr}\end{array}$ & $\mathrm{H}^{+}+\mathrm{Al}^{3+}$ & $\begin{array}{l}\text { MOS } \\
\mathrm{g} \mathrm{kg}^{-1}\end{array}$ \\
\hline \multicolumn{8}{|c|}{15 (Floresta primária) $^{(1)}$} \\
\hline $0-10 \mathrm{~cm}$ & 4,0 & 2,0 & 43,0 & 0,04 & 0,08 & 11,5 & 43,9 \\
\hline $11-30 \mathrm{~cm}$ & 4,0 & 1,0 & 22,0 & 0,03 & 0,02 & 9,3 & 21,4 \\
\hline \multicolumn{8}{|c|}{10 (Floresta primária) $^{(1)}$} \\
\hline $0-10 \mathrm{~cm}$ & 4,0 & 1,0 & 21,0 & 0,03 & 0,06 & 10,8 & 49,2 \\
\hline $11-30 \mathrm{~cm}$ & 3,9 & 1,0 & 40,0 & 0,04 & 0,07 & 12,8 & 30 \\
\hline \multicolumn{8}{|c|}{21 (reflorestamento de cinco anos) } \\
\hline $0-10 \mathrm{~cm}$ & 6,6 & 1,0 & 30,0 & 2,13 & 0,17 & 3,0 & 9,2 \\
\hline $11-30 \mathrm{~cm}$ & 5,9 & 0,4 & 22,0 & 0,95 & 0,09 & 4,8 & 3,4 \\
\hline \multicolumn{8}{|c|}{18 (reflorestamento de dez anos) } \\
\hline $0-10 \mathrm{~cm}$ & 5,0 & 4,0 & 25,0 & 0,38 & 0,05 & 8,6 & 12,6 \\
\hline $11-30 \mathrm{~cm}$ & 5,1 & 0,4 & 20,0 & 0,21 & 0,01 & 8,3 & 5,7 \\
\hline \multicolumn{8}{|c|}{2 (reflorestamento de quinze anos) } \\
\hline $0-10 \mathrm{~cm}$ & 4,6 & 2,0 & 42,0 & 0,03 & 0,03 & 10,3 & 16,8 \\
\hline $11-30 \mathrm{~cm}$ & 4,9 & 0,4 & 23,0 & 0,02 & 0,04 & 7,8 & 5,8 \\
\hline Ribeiro et al. (1999) $)^{(2)}$ & $5,5-6,0$ & $8,1-12,0$ & $71-12$ & $2,4-4,0$ & $0,9-1,5$ & $5,0-9,0^{(2)}$ & $4,0-7,0$ \\
\hline
\end{tabular}

Tabela 3. Teores de $\mathrm{Al}^{3+}$ trocável e micronutrientes $(\mathrm{Cu}, \mathrm{Fe}, \mathrm{Mn}$ e $\mathrm{Zn}$ ) disponíveis no solo em áreas alteradas (clareiras) e da floresta primária na BOGPM. Município de Coari, AM

\begin{tabular}{|c|c|c|c|c|c|}
\hline Profundidade & $\begin{array}{c}\mathrm{Al}^{3+} \\
-\mathrm{cmol}_{\mathrm{c}} \mathrm{dm}^{-3}\end{array}$ & $\mathrm{Cu}$ & $\mathrm{Fe}$ & Mn & Zn \\
\hline \multicolumn{6}{|c|}{15 (Floresta primária) $^{(1)}$} \\
\hline $0-10$ & 3,89 & 0,22 & 217 & 1,44 & 0,85 \\
\hline $11-30$ & 3,54 & 0,31 & 233 & 1,33 & 0,54 \\
\hline \multicolumn{6}{|c|}{10 (Floresta primária) $^{(1)}$} \\
\hline $0-10$ & 4,93 & 0,31 & 200 & 0,91 & 0,52 \\
\hline $11-30$ & 4,42 & 0,37 & 84 & 42 & 0,37 \\
\hline \multicolumn{6}{|c|}{21 (reflorestamento de cinco anos) } \\
\hline $0-10$ & 1,55 & 0,63 & 92 & 1,51 & 1,31 \\
\hline $11-30$ & 3,35 & 0,2 & 49 & 0,5 & 0,44 \\
\hline \multicolumn{6}{|c|}{18 (reflorestamento de dez anos) } \\
\hline $0-10$ & 4,53 & 0,21 & 21 & 0,26 & 0,44 \\
\hline $11-30$ & 5,62 & 0,25 & 9 & 0,21 & 0,37 \\
\hline \multicolumn{6}{|c|}{22 (reflorestamento de quinze anos) } \\
\hline $0-10$ & 6,68 & 0,3 & 45 & 0,17 & 0,69 \\
\hline $11-30$ & 6,2 & 0,31 & 14 & 0,13 & 0,4 \\
\hline Ribeiro et al. (1999) ${ }^{(2)}$ & $1,0-2,0^{(2)}$ & $1,3-1,8$ & $31-45$ & 0,9 a 1,2 & $1,6-2,2$ \\
\hline
\end{tabular}


retirada da floresta primária na Amazônia são apenas minimizados com o reflorestamento das áreas, mesmo com planta adaptadas as condições edafoclimáticas estudadas.

Utilizando ainda, o valor $50 \%$ na saturação por bases (V) como adequadas para plantas de baixa exigência nutricional (Raij, 1997), nas clareiras 5, $10 \mathrm{e}$ 15 anos seriam necessários, além das duas toneladas por hectare de calcário utilizada no plantio, a suplementação de $0,16,2,13$ e 2,66 t ha ${ }^{-1}$ do mesmo corretivo para alcançar o V\% almejado e repor as necessidades das plantas. Em solo com elevada acidez potencial, o principal efeito da aplicação de corretivos da acidez do solo é reduzir a atividade do $\mathrm{Al}^{3+}$ trocável (Raij, 1991; Havlin et al., 1999).

Mesmo o reflorestamento tendo sido feito com plantas oriundas da região, adaptadas a solos de baixa fertilidade natural (Rodrigues et al., 2006), a aplicação de $0,2 \mathrm{~kg}$ do formulado $10-30-10\left(\mathrm{~N}-\mathrm{P}_{2} \mathrm{O}_{5}-\mathrm{K}_{2} \mathrm{O}\right)$ num raio de $50 \mathrm{~cm}$ ao redor das plantas somente no plantio não foi suficiente para suprir as exigências nutricionais por vários anos, o que indica a necessidade de adubação de cobertura apara acelerar a regeneração das clareiras. O desbalanço e/ou carência nutricional podem ter acarretado em sintomas visuais de deficiências de $\mathrm{N}$ e $\mathrm{K}$ em todas as plantas, independente da espécie e idade de plantio.

O manejo da adubação feita com aplicação total dos fertilizantes somente na época de plantio nas condições edafoclimáticas estudadas com alta pluviosidade (Arruda, 2005) apresentou baixa eficiência, indicando a necessidade de parcelamento até o estabelecimento pleno das plantas. Tal resultado, sugere que devido o desenvolvimento vegetativo inicial lento característico das plantas da região e ao alto pluviométrico local (>2000 mm ano ${ }^{-1}$ ), que acarreta na lixiviação de nutrientes no perfil do solo, principalmente dos mais solúveis, como o $\mathrm{N}$ e $\mathrm{K}$.

Em locais com condições climáticas do ecossistema amazônico, Müller et al. (2000) em sistemas de consórcio de espécies florestais, e palmeiras usadas para recuperação de áreas desmatadas no município de Santa Izabel, Estado do Pará, também verificaram que além do calcário seria necessária a aplicação de fertilizantes no plantio: a) espécies florestais: aplicar dois litros de esterco de galinha poedeira e $0,2 \mathrm{~kg}$ de superfosfato triplo - SFT $\left(40 \%\right.$ de $\left.\mathrm{P}_{2} \mathrm{O}_{5}\right)$; b) palmeiras e frutíferas: aplicar dois litros de esterco de galinha e $0,2 \mathrm{~kg}$ de SFT na cova e em cobertura no primeiro ano de $0,07 \mathrm{~kg}$ do formulado N-P-K (10-18-20 + 0,03 kg de cloreto de potássio - $\mathrm{KCl}\left(60 \%\right.$ de $\left.\mathrm{K}_{2} \mathrm{O}\right)$ misturado na projeção da copa, e no segundo ano o dobro dessas quantidades, ou seja, bem acima da adubação utilizada na recuperação das clareiras.

Os atributos químicos do solo, mesmo utilizando as recomendações descritas por Müller et al. (2000), seriam necessários alguns ajustes para obter maior eficiência na adubação para os distróficos da Amazônia, como a substituição do SFT $(42 \pm 2 \%$ de $\mathrm{P}_{2} \mathrm{O}_{5}$ ) por superfosfato simples $\left(18 \%\right.$ de $\left.\mathrm{P}_{2} \mathrm{O}_{5}\right)$, visto ser fonte de $\mathrm{P}, \mathrm{Ca}$ e enxofre (S).

A elevação dos teores de MOS (Tabela 2) não proporcionou aumento na disponibilidade de micronutrientes (Tabela 3). A exceção do Fe disponível, os teores de $\mathrm{Cu}, \mathrm{Mn}$ e $\mathrm{Zn}$ disponível foram semelhantes aos obtidos por Moreira et al. (2009) no mesmo tipo de solo e ficaram abaixo das faixas consideradas adequadas por Ribeiro et al. (1999) com o extrator Mehlich 1 (Tabela 3). Apesar do aumento das quantidades de MO nas áreas de clareiras, os teores médios das duas florestas primárias $\left(46,6 \mathrm{~g} \mathrm{~kg}^{-1}\right)$ na camada de 0-10 cm foram de 406,5\%, 298,8\% e $177,4 \%$ superiores as jazidas com 5, 10 e 15 anos de plantio, respectivamente.

Tais resultados concordam com Moreira e Costa (2004), que nas mesmas condições edafoclimáticas, verificaram que a alteração do ambiente ocasionada pela retirada da camada superficial do solo alterou o teor de $\mathrm{MO}$ e os atributos químicos do solo. Isso sugere a necessidade de maior aporte inicial de fertilizantes químicos (minerais) e/ou orgânicos na regeneração das clareiras, restituindo o mais rápido possível a fertilidade do solo (Tabelas 1, 2 e 3).

A mudança da cobertura vegetal e profundidade de solo amostrada, mesmo com aplicação de calcário e $\mathrm{MgSO}_{4}$, acarretaram em variações no balanço de íons na CTC, ficando abaixo dos valores gerais tidos como adequados de $\mathrm{K}^{+}$(Tabela 4), que devem estar entre

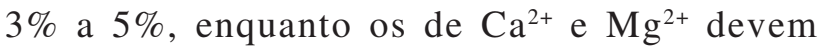
apresentar de $50 \%$ a $70 \%$ e de $10 \%$ a $15 \%$ da CTC, respectivamente (Tomé Júnior, 1997). Devido à baixa disponibilidade de $\mathrm{K}^{+}, \mathrm{Ca}^{2+}$ e $\mathrm{Mg}^{2+}$ no solo (Tabelas 1 e 2), as relações $\mathrm{Ca}^{2+} / \mathrm{Mg}^{2+}, \mathrm{Ca}^{2+} / \mathrm{K}^{+}, \mathrm{Mg}^{2+} / \mathrm{K}^{+}$e $\mathrm{K}^{+} /$ $\left(\mathrm{Ca}^{2+}+\mathrm{Mg}^{2+}\right)$ foram elevadas (Tabela 4$)$, indicando que 
Tabela 4. Balanço dos íons $\mathrm{K}^{+}, \mathrm{Ca}^{2+}$ e $\mathrm{Mg}^{2+}$ na CTC e relações $\mathrm{Ca}^{2+} / \mathrm{Mg}^{2+}, \mathrm{Ca}^{2+} / \mathrm{K}^{+}, \mathrm{Mg}^{2+} / \mathrm{K}^{+} \mathrm{e} \mathrm{K}^{+} /\left(\mathrm{Ca}^{2+}+\mathrm{Mg}^{2+}\right)$ na porcentagem de saturação por cátions da CTC nas clareiras e na floresta primária em diferentes profundidades.

\begin{tabular}{|c|c|c|c|c|c|c|c|}
\hline \multirow{2}{*}{$\begin{array}{l}\text { Profundidades } \\
\mathrm{cm}\end{array}$} & \multicolumn{3}{|c|}{ Balanço de íons } & \multirow[t]{2}{*}{$\mathrm{Ca}^{2+} / \mathrm{Mg}^{+}$} & \multirow[t]{2}{*}{$\mathrm{Ca}^{2+} / \mathrm{K}^{+}$} & \multirow[t]{2}{*}{$\mathrm{Mg}^{2+} / \mathrm{K}^{+}$} & \multirow{2}{*}{$\begin{array}{c}\mathrm{K}^{+} / \\
\left(\mathrm{Ca}^{2+}+\mathrm{Mg}^{2+}\right)\end{array}$} \\
\hline & $\mathrm{K}^{+(1)}$ & $\begin{array}{c}\mathrm{Ca}^{2+} \\
---\% \\
\end{array}$ & $\mathrm{Mg}^{2+}$ & & & & \\
\hline \multicolumn{8}{|c|}{15 (Floresta primária) $^{(2)}$} \\
\hline $0-10 \mathrm{~cm}$ & 1,27 & 0,34 & 0,69 & 0,50 & 0,27 & 0,54 & 1,23 \\
\hline & 0,65 & 0,26 & 0,17 & 1,50 & 0,40 & 0,27 & 1,51 \\
\hline \multicolumn{8}{|c|}{10 (Floresta primária) $^{(2)}$} \\
\hline $0-10 \mathrm{~cm}$ & 0,62 & 0,26 & 0,52 & 0,50 & 0,42 & 0,83 & 0,80 \\
\hline & 1,18 & 0,34 & 0,60 & 0,57 & 0,29 & 0,51 & 1,25 \\
\hline \multicolumn{8}{|c|}{21 (reflorestamento de cinco anos) } \\
\hline $0-10 \mathrm{~cm}$ & 1,42 & 39,66 & 3,16 & 12,53 & 20,70 & 1,65 & 0,04 \\
\hline & 0,94 & 15,99 & 1,51 & 1,06 & 1,26 & 1,19 & 0,41 \\
\hline \multicolumn{8}{|c|}{18 (reflorestamento de dez anos) } \\
\hline $\begin{array}{l}0-10 \mathrm{~cm} \\
11-30 \mathrm{~cm}\end{array}$ & 0,70 & 4,18 & 0,55 & 7,60 & 4,44 & 0,58 & 0,20 \\
\hline & 0,60 & 2,46 & 0,12 & 21,0 & 3,07 & 0,15 & 0,31 \\
\hline \multicolumn{8}{|c|}{22 (reflorestamento de quinze anos) } \\
\hline $0-10 \mathrm{~cm}$ & 1,02 & 0,29 & 0,29 & 1,00 & 0,21 & 0,21 & 2,40 \\
\hline & 0,74 & 0,51 & 0,51 & 0,50 & 0,25 & 0,51 & 1,31 \\
\hline Média 0-10 & 1,01 & 8,95 & 1,04 & 4,43 & 5,21 & 0,76 & 0,93 \\
\hline Média 11-30 & 0,82 & 3,91 & 0,58 & 4,93 & 1,05 & 0,53 & 0,96 \\
\hline Média geral & 0,85 & 2,41 & 0,53 & 4,68 & 3,13 & 0,64 & 0,95 \\
\hline
\end{tabular}

${ }^{(1)}$ Extrator Mehlich 1 (K disponível) e $\mathrm{KCl} 1,0 \mathrm{~mol} \mathrm{~L}^{-1}\left(\mathrm{Ca}^{2+}\right.$ e $\mathrm{Mg}^{2+}$ trocável). Saturação de $\mathrm{K}=\left(\mathrm{K}^{+} / \mathrm{CTC}\right) \times 100$; saturação de $\mathrm{Ca}$ $=\left(\mathrm{Ca}^{2+} / \mathrm{CTC}\right) \times 100 ;$ saturação de magnésio $=\left(\mathrm{Mg}^{2+} / \mathrm{CTC}\right) \times 100 .{ }^{(2)}$ Áreas adjacentes as clareiras.

se for feita uma adubação equilibrada, essas relações podem ser mantidas, aumentando a eficiência de uso desses nutrientes.

\section{Conclusões}

O manejo adotado na revegetação das clareiras nas condições edafoclimáticas da Amazônia Central indicam que a manutenção da fertilidade do solo está diretamente associada ao teor de matéria orgânica e somente a prática adubação realizada por ocasião do plantio nas áreas de clareiras não foi suficiente para elevar os teores disponíveis aos níveis de floresta primária.

\section{Agradecimentos}

À FINEP/Petrobrás (Programa CTPetro), pelo suporte financeiro e apoio logístico para realização deste trabalho e a Embrapa Amazônia Ocidental pelas análises laboratoriais.

\section{Literatura Citada}

ARRUDA, M. R., et al. 2012. Produtividade, fertilidade do solo e estado nutricional de clones de guaranazeiro cultivados com fontes orgânicas de nutrientes. Revista de Ciências Agrárias (Brasil) 55:311-317.

ARRUDA, W. C. 2005. Estimativa dos processos erosivos na base de Operações Geólogo Pedro de Moura - URUCU - Coari - AM. Dissertação Mestrado. Manaus, AM, Universidade Federal do Amazonas, 80p.

COELHO, A. M. 2004. Agricultura de precisão: manejo da variabilidade espacial e temporal dos solos e das culturas. In: Curi, N., et al, eds. Tópicos em Ciência do Solo. Viçosa, MG, SBCS. pp.529-562.

DEMATTÊ, J. L. I. 1988. Manejo de solos ácidos dos trópicos úmidos - Região Amazônica. Campinas, SP, Fundação Cargill. 215p. 
EMPRESA BRASILEIRA DE PESQUISA AGROPECUARIA. 1997. Manual de métodos de análise de solo. Rio de Janeiro, RJ, EMBRAPA / CNPS. 212p.

FEARNSIDE, P. M. 1999. Biodiversity as an environmental service in Brazil's Amazonian forest: risks, value and conservation. Environment Conservation 26:305-321.

FREITAS, A. R., et al. 2008. Técnica de análises exploratórias em dados de cultivares de alfafa. Revista Brasileira de Zootecnia. 37:1531-1536.

HAVLIN, J. L., et al. 1999. Soil fertility and fertilizers, an introduction to nutrient management. Upper Saddle River, EUA, Prentice Hall. 499p.

LIMA FILHO, D. A., et al. 2001. Inventário florístico de floresta umbrófila densa de terra firme, na região do rio Urucu, Amazonas, Brasil. Acta Amazonica 31(4):565-579.

MALAVOLTA, E. 1987. Fertilidade dos solos da Amazônia. In Vieira, L. S.; Santos, P. C. T. C., eds. Amazônia: seus solos e outros recursos naturais. São Paulo, SP, Agronômica Ceres. pp.374-416.

MOREIRA, A.; COSTA, D. G. 2004. Dinâmica da matéria orgânica na recuperação de clareiras da floresta amazônica. Pesquisa Agropecuária Brasileira 39(10):1013-1020.

MOREIRA, A.; FAGERIA, N. K. 2009. Soil chemical attributes of Amazonas State, Brazil. Communications in Soil Science and Plant Analysis 40(17/18):2912-2925.

MOREIRA, A.; GONÇALVES, J. R. P. 2006. Available Phosphorus and Potassium Status of Soils of Amazonas State. Better Crops 90(1):30-32.

MOREIRA, A; TEIXEIRA, W. G.; MARTINS, G. C. 2009. Extratores e disponibilidade de micronutrientes em Terra Preta do Índio da Amazônia central (Brasil). Ciencia del Suelo (Argentina) 27:127-134.

MÜLLER, C. H., et al. 2000. Sistemas de consórcio envolvendo o cupuaçuzeiro (Theobroma grandiflorum) como cultura principal. In Congresso Brasileiro de Sistemas Agroflorestais. Manaus, AM, EMBRAPA. pp.306-309.

PEZARICO, C. R., et al. 2013. Indicadores de qualidade do solo em sistemas agroflorestais. Revista de Ciências Agrárias 56:40-47.

POGGIANI, F.; SCHUMACHER, M. V. 2004. Nutrient cycling in native forest. In: Gonçalves, J. L. M.; Benedetti, V. eds. Forest and fertilization. Piracicaba, SP, IPEF. pp.285-306.

PRUDENTE, A. L. C., et al. 2010. Serpentes da Bacia petrolífera de Urucu, Município de Coari, Amazonas, Brasil. Acta Amazonica, 40:381-386.

RAIJ, B. van. 1991. Fertilidade do solo e adubação. Piracicaba, SP, Potafós/Ceres. 343p.

RAIJ, B. van et al. 1997. Recomendações de adubação e calagem para o Estado de São Paulo. Campinas, SP, Instituto Agronômico de Campinas. 285p.

RIBEIRO, A. C.; GUIMARÃES, P. T. G.; ALVARES VENEGAS, V. H. 1999. Recomendação para o uso de corretivos e fertilizantes em Minas Gerais, $5^{\text {a }}$ Aproximação. Viçosa, MG, SFSEMG. 359p.

RODRIGUES, M. R. L.; BARROS, M. E.; TEIXEIRA, W. G. 2006. Produção de mudas florestais para plantio em áreas degradadas da província petrolífera de Urucu, Coari - Amazonas. In Workshop de Avaliação Técnica e Científica. Manaus, AM, CTPetro Amazônia. 4p.

SANCHEZ, P. A.; SALINAS, J. G. 1981. Low input management technology for managing Oxisols and Utisols in tropical America. Advances in Agronomy 34:279-406.

SANTOS JÚNIOR, U. M.; GONÇALVES, J. F. C.; FELPAUSCH, T. R. 2006. Growth, leaf nutrient concentration and photosynthetic nutrient use efficiency in tropical tree species planted in degraded areas in central Amazonia. Forest Ecology and Management 226:299-309.

STARK, N. 1970. Nutrient cycling: nutrient distribution in some Amazonian soils. Tropical Ecology 12(1):24-50.

TOMÉ JUNIOR, J. B. 1997. Manual para interpretação de análise de solo. Guaíba, RS, Editora Agropecuária. 247p.

UCHOA, J. I., et al. 2006. Caracterização química dos compostos orgânicos produzidos na Estação de Tratamentos de Resíduos da Base Petrolífera de Urucu. In Workshop de Avaliação Técnica e Científica Manaus, AM, CTPetro Amazônia. 4p.

VIEIRA, L. S.; SANTOS, P. C. T. C. 1987. Amazônia: seus solos e outros recursos naturais. São Paulo, SP, Agronômica Ceres. 416p 